\title{
Effects of band filling in the Anderson-Falicov-Kimball model
}

\author{
Rubens D. B. Carvalho and M. A. Gusmão \\ Instituto de Física, Universidade Federal do Rio Grande do Sul, C.P. 15051, 91501-970 Porto Alegre, Brazil
}

(Received 12 November 2012; published 19 February 2013)

\begin{abstract}
In this work, we study the Anderson-Falicov-Kimball model within the dynamical mean field theory for the Bethe lattice, restricting our analysis to the nonmagnetic case. The one-particle density of states is obtained by both arithmetic and geometric averages over disorder, since only the latter can detect localization in the absence of an energy gap. Varying the strengths of Coulomb interaction and disorder at zero temperature, we construct phase diagrams for this model, where we distinguish spectral regions with localized states, with extended states, or with a correlation-induced gap. With this, we identify metal-insulator transitions driven by correlation and disorder, as well as the competition between these effects. This is done for various band fillings, since our main interest here is to study how the variation of the electron density affects the phase diagrams previously obtained for half-filling. The picture revealed by the density of states is further checked by evaluating the static and dynamic conductivities, including temperature effects.
\end{abstract}

DOI: 10.1103/PhysRevB.87.085122

PACS number(s): 71.10.Fd, 71.23.-k, 71.30.+h

\section{INTRODUCTION}

Electrons in narrowband solids are strongly affected by the Coulomb interaction as well as lattice disorder. Both effects can lead to a metal-insulator transition (MIT), but of different nature: a correlation-induced Mott insulating state ${ }^{1,2}$ and disorder-induced Anderson localization. ${ }^{3}$ The existence of the Mott phase is also significantly dependent on band filling, and one can expect the interplay between correlations and disorder to be substantially affected by variations of the number of electrons.

Theoretical approaches to deal with this kind of system are usually based on the Hubbard Hamiltonian or related models, including disorder as a distribution of on-site energies. The Mott-Hubbard MIT is characterized by a gap opening at the Fermi level in the one-particle density of states (DOS) ${ }^{2}$ Hence, it seems appropriate to utilize the DOS at the Fermi level as a monitoring parameter of an MIT. However, in the disordered case one has to average the DOS over disorder. As it happens, the simple (arithmetic) averaged DOS does not show any gap at the Anderson localization transition. It is the typical value of the DOS, obtained by means of a geometric average over disorder ${ }^{4}$ (with possible extensions involving generalized averages ${ }^{5}$ ), that does go to zero when the states at the Fermi level become localized, and thus can be used as indicative of the Anderson transition. Nevertheless, the information provided by the arithmetic average is also important in that it keeps track of all the states. Thus, the combined information from both averages of the DOS in the whole energy range allows one to locate the regions of localized and extended states.

Apart from the effect of disorder, one of the most employed approaches to the problem of determining electronic structure in strongly correlated systems is the dynamical mean field theory (DMFT), introduced by Metzner and Vollhardt ${ }^{6}$ in 1989 (for a review, see Refs. 7 and 8). The theory is formally exact in the limit of infinite dimensions, in the sense that in this limit the self-energy becomes purely local, allowing for an exact self-consistent connection between the site-diagonal one-particle Green's function of the lattice and that of an effective single-site (or impurity) problem. Nevertheless, the latter has no closed analytical solution in general, and one must resort to approximate impurity solvers, mostly numerical. ${ }^{9}$ Alternatively, one can use "simplified" models that allow for closed analytic relations which can be solved by a simple numerical iteration procedure. This is the case of the simplified Hubbard model, as introduced by van Dongen and Vollhardt. ${ }^{10}$ It is a reinterpretation of the Falicov-Kimball (FK) model $^{11}$ in which the two spinless fermions of the latter represent the two spin states of the electrons, thus eliminating spin-flip processes. The FK model has an exact solution in infinite dimensions, ${ }^{12-14}$ and it has been used with relative success ${ }^{15-17}$ to obtain phase diagrams in the presence of local disorder, characterizing the Anderson-Falicov-Kimball (AFK) model.

Essentially all the previous studies of correlation and disorder in the the Hubbard or FK models ${ }^{15-17}$ were restricted to the half-filled case. The obtained phase diagrams for varying disorder strength and Coulomb interaction show a metallic state, a Mott-insulator phase (which might be hidden by antiferromagnetic order), and an Anderson-localization regime. Here we focus on the AFK model away from halffilling, in the nonmagnetic case. We follow the evolution of the phase diagram from the strongly correlated regime near half-filling, where the Mott-insulator phase is suppressed by doping, toward the uncorrelated Anderson-localization limit at very low filling. We will also discuss the characteristics of the metallic regimes as probed by the optical and static conductivities.

The paper is organized as follows. In Sec. II we present the model and the DMFT approach to solve it, including the disorder averages of the DOS and calculation of conductivity. Section III describes our results for the DOS at various band fillings, typical values of the Coulomb interaction $U$, and a broad range of values of disorder strength $\Delta$. It also includes spectral diagrams showing the frequency regions with extended or localized states as $U$ and $\Delta$ are varied, and $\Delta$ versus $U$ phase diagrams for different filling fractions. Complementary results for the conductivity (both static and frequency-dependent) are shown in Sec. IV, including its behavior with temperature. Final comments and conclusions are presented in Sec. V. 


\section{MODEL AND METHOD}

The Anderson-Falicov-Kimball model can be represented by the Hamiltonian

$$
H=\sum_{i} \varepsilon_{i}\left(n_{i}^{c}+n_{i}^{f}\right)-t \sum_{\langle i j\rangle} c_{i}^{\dagger} c_{j}+U \sum_{i} n_{i}^{c} n_{i}^{f},
$$

where we employ the usual notation of creation, annihilation, and number operators (in the Wannier representation) for two kinds of spinless fermions. The $c$ fermions can move with a nearest-neighbor hopping integral $t$, and experience Coulomb repulsion $U$ by the nonmoving $f$ fermions. The local energies $\varepsilon_{i}$ are random, with a uniform probability distribution of width $\Delta$.

The exact solution of the FK model in infinite dimensions $^{12-14}$ is an example of DMFT with an analytic solution of the impurity problem. It yields a closed form for the $c$-particle Green's function at a finite temperature $T$,

$$
G_{i i}\left(\omega_{n}\right)=\frac{1-p}{i \omega_{n}-\bar{\varepsilon}_{i}-\lambda\left(\omega_{n}\right)}+\frac{p}{i \omega_{n}-\bar{\varepsilon}_{i}-U-\lambda\left(\omega_{n}\right)},
$$

where $\omega_{n}=(2 n+1) \pi T$, for integer $n$, are Matsubara frequencies, $\bar{\varepsilon}_{i} \equiv \varepsilon_{i}-\mu$ is the local energy measured with respect to the chemical potential, $p$ measures the probability of finding an $f$ particle at site $i$, and $\lambda\left(\omega_{n}\right)$ are the Fourier components of the dynamical mean field connecting this site to the $c$-particle reservoir that replaces the lattice.

Clearly, $p$ can be viewed as the average number of $f$ particles per site, and must be determined self-consistently. Keeping in mind the analogy of $c$ and $f$ fermions with spin-up and spin-down electrons in the Hubbard model, the total number of particles per site corresponds to the band-filling fraction $n$. At half-filling $(n=1)$ and low temperatures, an antiferromagnetic phase is known to be stable, ${ }^{13}$ corresponding to a chess-board-like distribution of $c$ and $f$ particles on a bipartite lattice. This ordered state is stable in the presence of Anderson-like disorder ${ }^{17}$ up to a critical disorder strength $\Delta_{c}$ which depends on the value of $U$. On the other hand, a paramagnetic state must have equal average numbers for both particles, i.e., $\left\langle n_{c}\right\rangle=\left\langle n_{f}\right\rangle$, independent of the site index. Since we are interested in the effect of band filling, and since the magnetic state tends to be suppressed away from half-filling, we will focus on the nonmagnetic case, for which the self-consistent solution implies that $p=n / 2$. Then the only unknown in Eq. (2) is the dynamical mean field $\lambda\left(\omega_{n}\right)$, which can be determined from the self-consistent condition that the site-diagonal lattice Green's function must be equal to the effective single-site solution with the same self-energy $\Sigma_{i}\left(\omega_{n}\right)$, which is purely local in the limit $d \rightarrow \infty$. This can be expressed by the equality

$$
\int \frac{\rho_{0}(\epsilon) d \epsilon}{i \omega_{n}-\bar{\varepsilon}_{i}-\epsilon-\Sigma_{i}\left(\omega_{n}\right)}=\frac{1}{i \omega_{n}-\bar{\varepsilon}_{i}-\lambda\left(\omega_{n}\right)-\Sigma_{i}\left(\omega_{n}\right)},
$$

$\rho_{0}(\varepsilon)$ being the density of states (DOS) for the uncorrelated band. The right-hand side of this last equation reproduces the local Green's function (2) if we write the self-energy as

$$
\Sigma_{i}\left(\omega_{n}\right)=p U+\frac{p(1-p) U^{2}}{\omega-\bar{\varepsilon}_{i}+\mu-(1-p) U-\lambda\left(\omega_{n}\right)} .
$$

With disorder, the Green's functions must be averaged over the distribution of local energies $\varepsilon_{i}$. Then, Eq. (3) implies that

$$
G_{i i}^{\mathrm{av}}\left(\omega_{n}\right)=\int \frac{\rho_{0}(\epsilon) d \epsilon}{\left[G_{i i}^{\mathrm{av}}\left(\omega_{n}\right)\right]^{-1}+\lambda\left(\omega_{n}\right)-\epsilon},
$$

complemented by the disorder average of Eq. (2). Actually, we have to determine the single-site spectral density $\rho_{i}(\omega)$, for each disorder realization, by performing the analytic continuation $i \omega_{n} \rightarrow \omega+i 0^{+}$to obtain the retarded Green's function $G_{i i}(\omega)$, which yields $\rho_{i}(\omega)=-\operatorname{Im} G_{i i}(\omega) / \pi$. The latter is then averaged to obtain the final spectral density, or local DOS.

At this point, it is worth recalling that we must use two kinds of averages, arithmetic and geometric. With $[\cdots]_{a}$ denoting the arithmetic average, we have $\rho_{a}(\omega)=\left[\rho_{i}(\omega)\right]_{a}$ and $\rho_{g}(\omega)=\exp \left[\ln \rho_{i}(\omega)\right]_{a}$. The self-consistent determination of the corresponding $G_{a}(\omega)$ or $G_{g}(\omega)$ implies that we have different hybridization functions $\lambda_{a}(\omega)$ and $\lambda_{g}(\omega)$.

We will consider an uncorrelated DOS corresponding to a Bethe lattice $^{18}$ in the limit of infinite coordination $(z \rightarrow \infty)$, $\rho_{0}(\epsilon)=\frac{4}{\pi W} \sqrt{1-4(\epsilon / W)^{2}}$, where $W=4 t^{*}$ is the bandwidth, $t^{*}=\sqrt{z} t$ being the scaled hopping integral. ${ }^{19}$ Since no hopping loops are possible in the Bethe lattice, the only way for an electron to visit the rest of the lattice is by going out and coming back through the same nearest-neighbor site (any one of them). This implies a simple relation for the hybridization function, $\lambda_{\alpha}(\omega)=z t^{2} G_{\alpha}(\omega)=W^{2} G_{\alpha}(\omega) / 16$, where $\alpha=a, g$. In the following, we will choose the bandwidth as the unit of energy, so that $W=1$. In addition, we will adopt the usual convention of dropping the Planck and Boltzmann constants, so that energy, temperature, and frequency are all measured in the same units, and will be given here by pure numbers.

\section{DOS AND PHASE DIAGRAMS}

To perform a detailed analysis of the problem, we solved numerically the DMFT equations, obtaining densities of states for a variety of values of the relevant parameters. All the calculations reported in this section are for $T=0$. The most probable value of the DOS is used to monitor the metal-insulator transition mediated by correlation and/or disorder. Both $\rho_{g}(\omega)$ and $\rho_{a}(\omega)$ are used to distinguish between spectral regions with a gap, with extended states, or with localized states.

The phase diagrams previously obtained ${ }^{16,17}$ for the halffilling case without magnetic order allow us to distinguish three different regimes: (i) the weak-interaction regime for $U<1 / 2$, where one sees Anderson localization as $\Delta$ becomes sufficiently large, but there are no significant correlation effects; (ii) the intermediate-interaction regime for $1 / 2<U \lesssim$ 1.4, where Mott-insulator, metallic, and Anderson-localization regions exist for varying $\Delta$; and (iii) the strong-interaction regime for $U \gtrsim 1.4$, where no metallic state exists, but the insulating state shows a competition between correlation and disorder effects. For our analysis, we will choose a typical value of $U$ in each of these regimes, namely $U=0.3,0.9$, and 1.5. We will then determine the averaged densities of states for five band fillings, ranging from $n=0.2$ to 1.0.

In the first regime $(U=0.3)$, Fig. 1 shows densities of states with arithmetic and geometric average for a moderate 

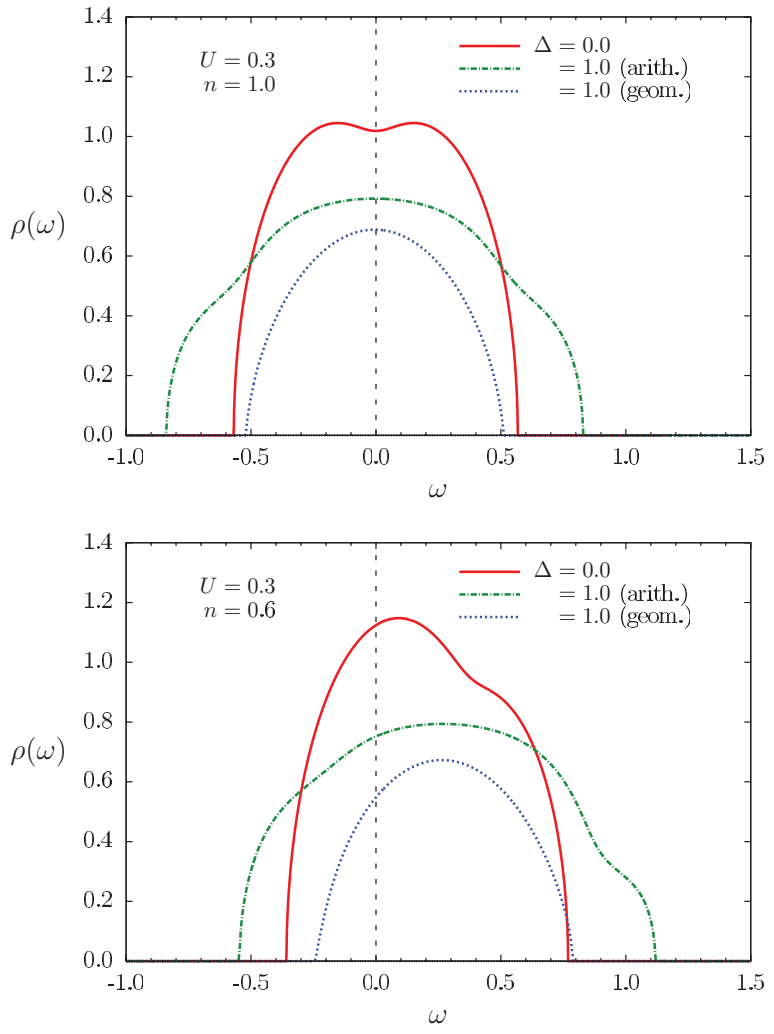

FIG. 1. (Color online) Local DOS at half-filling (top) and at $n=$ 0.6 (bottom) for $U=0.3$, showing the effect of a moderate disorder ( $\Delta=1.0$ ) with both kinds of averages. Notice the reduction of the number of extended states (given by the geometric average) and the overall band broadening (shown by the arithmetic average).

disorder $(\Delta=1.0)$ in comparison with the clean limit. For this low interaction value there is no Mott gap, only a shallow depletion of the DOS at the Fermi level for the half-filled band in the clean limit. The main difference when moving away from half-filling is the loss of particle-hole symmetry, with the Fermi level being displaced towards the band bottom. The general behavior of the two kinds of averages is clearly noticeable in Fig. 1, that is, the arithmetic-averaged DOS is broadened but preserves the area, while the geometric-averaged one loses spectral weight due to a reduction in the number of extended states. It is then clear that the states at the band edges are the first to become localized.

This situation does not change qualitatively when further reducing the band filling, as can be seen from the spectral diagrams shown in Fig. 2. These diagrams show the spectral regions where there are extended or localized states in the band. Also clearly visible are the overall band broadening as well as the suppression of extended states above a critical disorder strength that characterizes the localization transition. Observing the dashed vertical line that marks the Fermi level, one can see that Anderson localization occurs at different values of $\Delta$ for different band fillings.

In the intermediate-interaction regime we observe the opening of a Mott gap, as shown in the top panel of Fig. 3. This gap shrinks with increasing disorder and eventually closes. It happens first for the arithmetic-averaged DOS, implying that the gap observed in the geometric average is actually filled
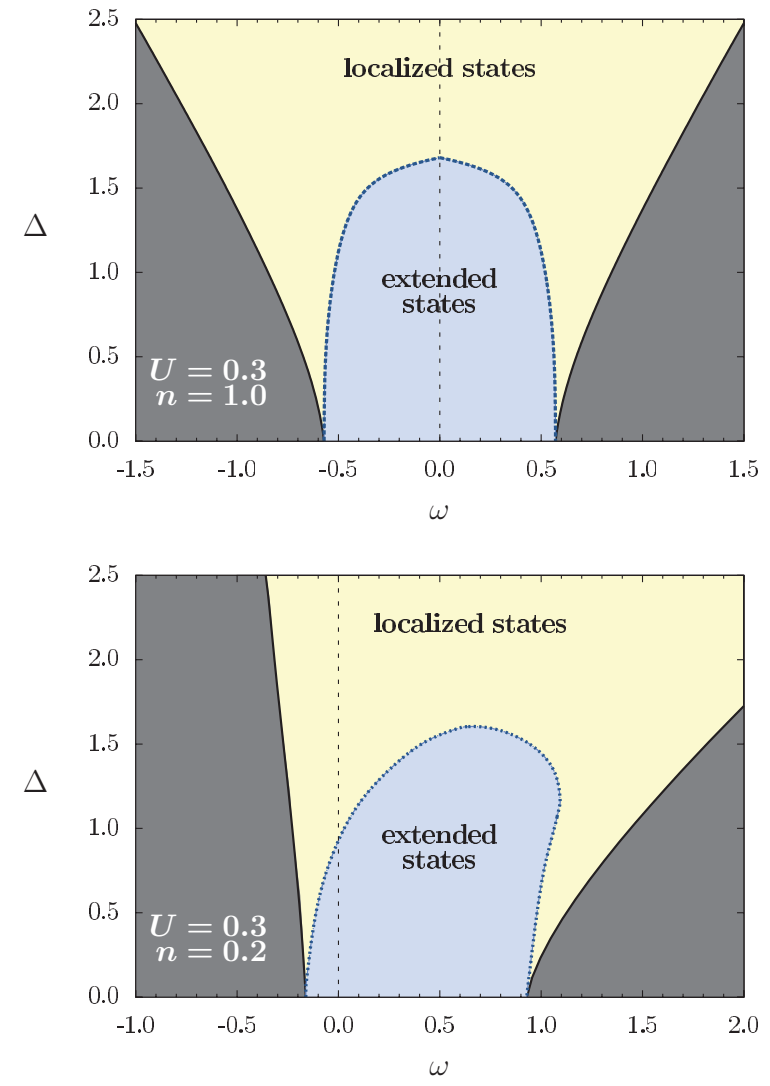

FIG. 2. (Color online) Spectral diagrams for $U=0.3$ at halffilling (top) and very low filling (bottom). No qualitative changes are noticed, except for the loss of particle-hole symmetry and the displacement of the Fermi level toward the band's lower edge when the band filling is reduced.

with localized states. Away from half-filling (bottom panel of Fig. 3), the Fermi level drops into the lower Hubbard subband and the system becomes metallic. This is confirmed by the spectral diagrams of Fig. 4. There we can see that the topology of the spectral diagrams changes as the band filling is reduced. The half-filled case (not shown) is similar to the top panel of Fig. 4, but it shows particle-hole symmetry, so that the gap at weak disorder contains the Fermi level. Then, with increasing disorder the system evolves from a Mott insulator, through a regime in which the Mott gap is filled with localized states (corresponding to the DOS in the top panel of Fig. 3), into a metallic state (when the gap in the geometric-averaged DOS also closes), finally becoming an Anderson insulator when all the states localize.

For the strong-interaction regime, shown for $U=1.5$ in Fig. 5, we see a more robust correlation gap, as expected. At half-filling (top panel of Fig. 5), the low-disorder behavior is the same as in the intermediate regime, but it changes significantly for strong disorder. For instance, the Mott gap never closes in the region where extended states still exist, so that we have a direct transition between Mott and Anderson insulators. In contrast, for low band filling (bottom panel of Fig. 5), the low-energy physics is essentially the same for all correlation regimes, showing only the Anderson-localization transition from the metallic state, although the diagrams are very different on the higher-energy part of the spectrum. 

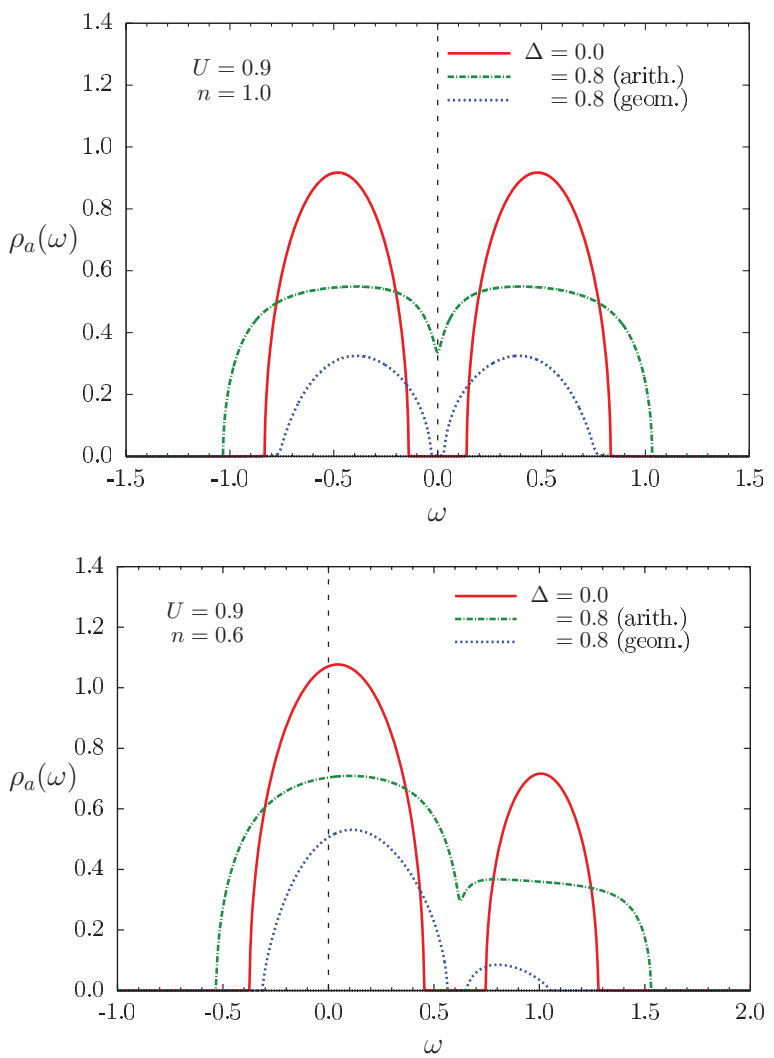

FIG. 3. (Color online) DOS for $n=1.0$ (top) and 0.6 (bottom), $U=0.9$, and $\Delta=0.8$, a disorder strength for which a gap still exists in the geometric average but no longer in the arithmetic one.

The above results can be summed up in phase diagrams of the Anderson-Falicov-Kimball model for varying $U$ and $\Delta$ at different band fillings, which are shown in Fig. 6. The lines for each $n$ correspond to the $\Delta$ values at which the Fermi level crosses the borderline between extended and localized states in the spectral diagrams for each $U$. The only exception is the point at $U=0.5$ and $\Delta=0$ at $n=1$, for which the transition is the Mott MIT. For all the other band fillings, as can be seen in Fig. 6, the Mott phase does not exist. Nevertheless, the Anderson MIT is clearly affected by correlation. Obviously, this effect is stronger near half-filling, and tends to disappear as the electron density becomes very low. At electron densities not far from $n=1$, we can see that the weak-disorder metallic state is quickly suppressed as the disorder increases. In this correlated Anderson-localization regime, the Fermi level falls in a region of localized states close to a correlation gap. As the disorder is further increased, we see a reentrance of the metallic state, now much less correlated (as will be better seen in Sec. IV), which is finally suppressed in the strong-disorder region, where the normal Anderson-insulator state sets in.

\section{CONDUCTIVITY}

The features that we observed in the spectral diagrams must show up in the optical conductivity, and it is interesting to study this quantity in detail. In general, the frequency-dependent conductivity $\sigma(v)$, in the context of linear-response theory, is described by the Kubo formula, which involves a currentcurrent correlation function. In DMFT, the calculation of this
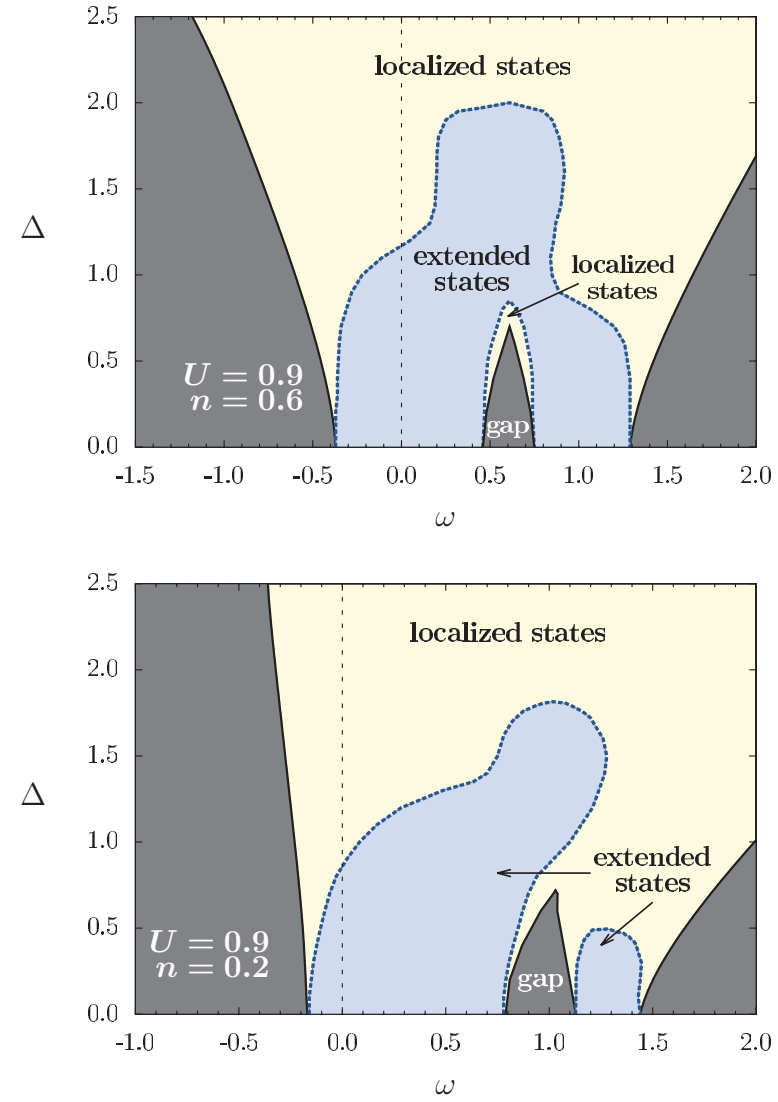

FIG. 4. (Color online) Spectral phase diagram for $U=0.9$, with $n=0.6$ (top) and 0.2 (bottom). The half-filling diagram is similar to the top panel, except that particle-hole symmetry is restored, so that the system is a Mott insulator for weak disorder.

function is simplified due to the absence of vertex corrections, which vanish in the limit $d \rightarrow \infty$. We then have $\mathrm{e}^{20}$

$$
\begin{aligned}
\sigma(v)= & \pi \int d \epsilon \rho_{0}(\epsilon) \int d \omega \rho(\epsilon, \omega) \rho(\epsilon, \omega+v) \\
& \times \frac{f(\omega)-f(\omega+v)}{v},
\end{aligned}
$$

where $f(\epsilon)$ is the Fermi-Dirac function, $\rho_{0}(\epsilon)$ is the uncorrelated DOS, and $\rho(\epsilon, \omega)$ is the spectral density of the correlated energy-dependent Green's function. From Eq. (5), after the analytic continuation $i \omega_{n} \rightarrow \omega+i 0^{+}$, we have

$$
\rho(\epsilon, \omega)=-\frac{1}{\pi} \operatorname{Im}\left\{\left[G_{i i}^{\mathrm{av}}(\omega)\right]^{-1}+\lambda(\omega)-\epsilon\right\}^{-1},
$$

and we must choose the geometric average, since only extended states contribute to the conductivity.

The limit $v \rightarrow 0$ in Eq. (6) gives us the static conductivity as a function of temperature,

$$
\sigma(T)=\frac{\pi}{T} \int d \epsilon \rho_{0}(\epsilon) \int d \omega[\rho(\epsilon, \omega)]^{2} f(\omega)[1-f(\omega)] .
$$

Results for the weak-interaction regime are shown in Fig. 7. The behavior is essentially what one should expect for a conductor, but the low-frequency peak is progressively reduced as disorder increases, and eventually disappears at the Anderson-localization transition, where all the states become 

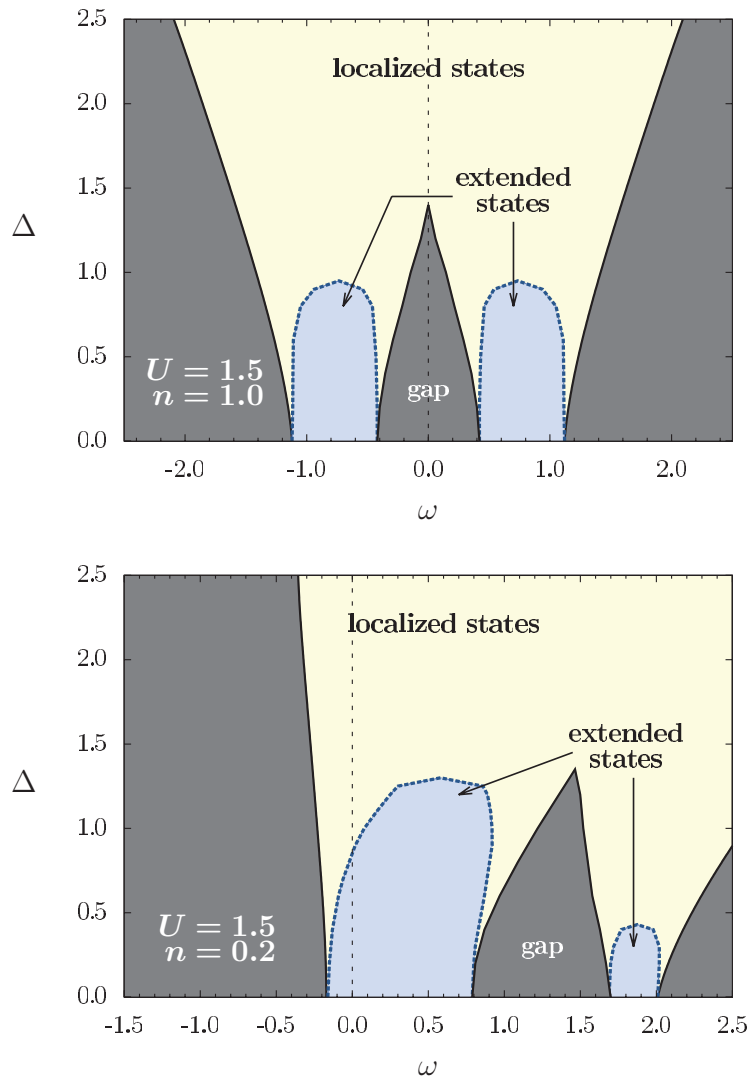

FIG. 5. (Color online) Spectral phase diagram in the strongcoupling regime ( $U=1.5$ ) for $n=1.0$ (top) and 0.2 (bottom).

localized and the conductivity vanishes. In the inset of the top panel of Fig. 7 we highlight the existence of thermal activation in the clean limit at half-filling, as we can see that the conductivity for $v \rightarrow 0$ grows with temperature. This can be understood by inspecting the corresponding DOS, shown in the top panel of Fig. 1, where it is clear that there are regions of higher DOS slightly away from the Fermi level. Both this feature of the DOS and the conductivity behavior at low frequencies are non-Fermi-liquid characteristics, as expected for the FK model due to the absence of spin-flip processes.

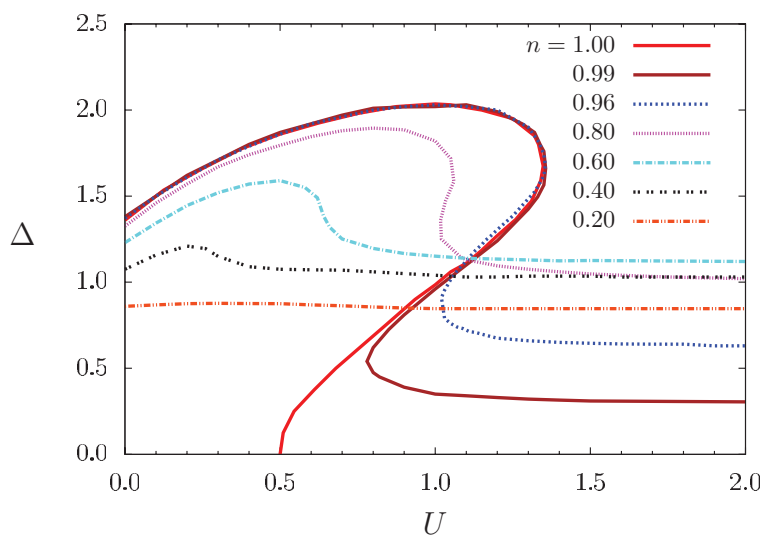

FIG. 6. (Color online) Phase diagram of the Anderson-FalicovKimball model for various concentrations. A Mott insulator phase exists only at half-filling.
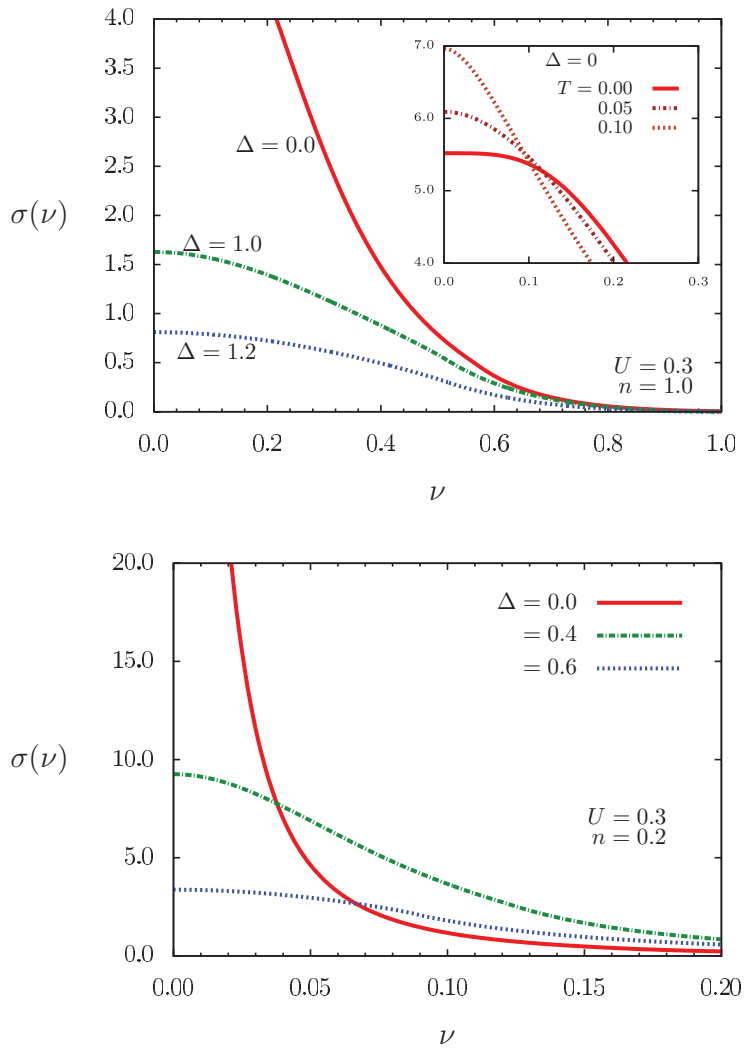

FIG. 7. (Color online) Optical conductivity in the weakinteraction regime $(U=0.3)$ at half-filling (top) and in the lowdensity limit $(n=0.2)$. In both cases, we see a typical conductor behavior, with a more pronounced Drude-like peak at low density and in the clean limit. The inset in the top panel shows an activation behavior at low frequencies for $n=1$ and no disorder, due to the lower DOS at the Fermi level observed in Fig. 1.

The low-density scenario does not change in the other interaction regimes, except for the overall scale and for the visibility of high-frequency "bumps" coming from the

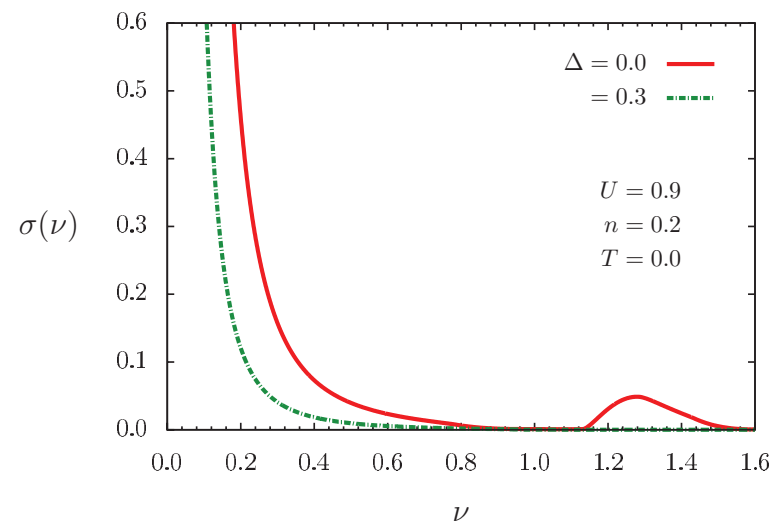

FIG. 8. (Color online) Optical conductivity in the intermediateinteraction regime $(U=0.9)$ in the low-density limit $(n=0.2)$. Apart from the Drude-like behavior, a nonzero conductivity appears in the clean limit at high frequencies due to extended states existing above the correlation gap. This is suppressed by a moderately low disorder. For much higher disorder (not shown), the low-frequency peak also vanishes and the system becomes an Anderson insulator. 


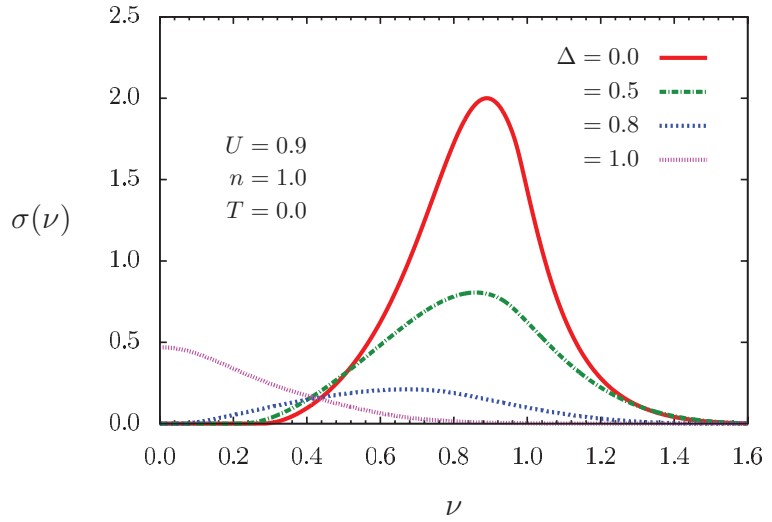

FIG. 9. (Color online) Optical conductivity in the intermediateinteraction regime $(U=0.9)$ at half-filling. The Mott gap is visible for weak disorder, but a metallic behavior is recovered for a certain range of values of $\Delta$ (exemplified by $\Delta=1.0$ ).

upper Hubbard subband, which quickly vanish with increasing disorder. This is exemplified for $U=0.9$ in Fig. 8, but it also occurs for $U=1.5$. One can easily correlate these conductivity features with the low-density spectral diagrams of Figs. 4 or 5 .

The situation is very different at half-filling, as we begin with a Mott insulator in the clean limit for intermediate or strong Coulomb interaction. Again we illustrate this with results for $U=0.9$, shown in Fig. 9. There we can see a progressive suppression of the high-frequency peak in the optical conductivity as disorder grows, and the appearance of a low-frequency one when the system becomes metallic. This metallic peak first grows, then diminishes, and finally vanishes at the critical disorder strength for Anderson localization. For this case, the sequence of states, from Mott-insulator to metal to Anderson insulator, can be followed along a vertical line at $U=0.9$ in the phase diagram for $n=1.0$ in Fig. 6. Notice that a low-frequency peak always exists away from half-filling, although only for very weak disorder as $n \rightarrow 1$.

We now turn to the effect of temperature on the conductivity. It is worth mentioning that this effect comes essentially from the Fermi functions appearing in Eqs. (6) and (8). The spectral functions are not affected by temperature, except for a very

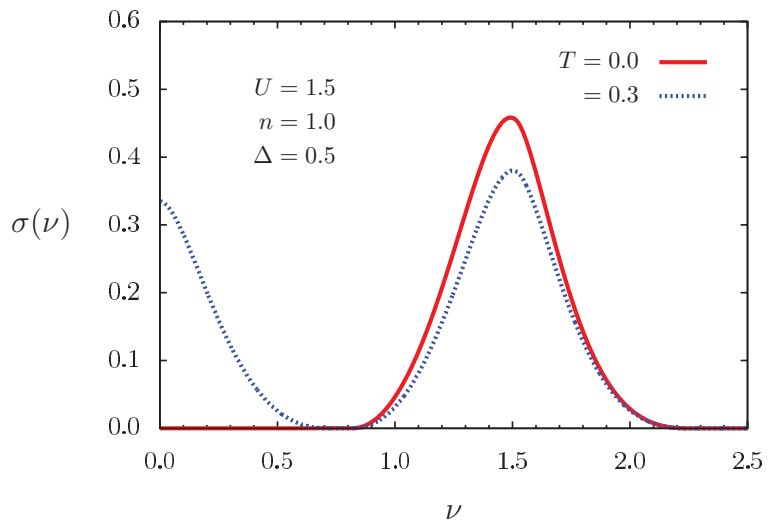

FIG. 10. (Color online) Effect of temperature on the optical conductivity for $U=1.5$ and $n=1$. A finite temperature yields a nonzero conductivity for frequencies inside the Mott gap.

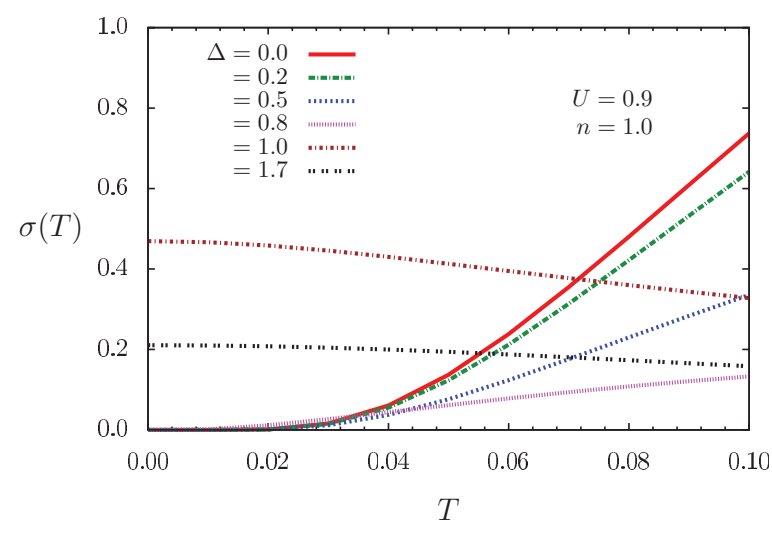

FIG. 11. (Color online) Static conductivity as a function of temperature for $U=0.9$ at half-filling. The activated conductivity in the insulating region at low disorder evolves to a metallic behavior as the disorder increases.

small displacement of the chemical potential, which does not occur at half-filling due to particle-hole symmetry.

The most striking thermal effect in the optical conductivity occurs for a Mott-insulator ground state, as illustrated in Fig. 10 for $U=1.5$ at half-filling. The temperature effect is to "turn on" the optical conductivity inside the Mott gap. Of course, the obtained intensity depends on the temperature and on the gap size. For the strong-coupling case shown in Fig. 10, the temperature necessary for such a visible effect is unphysically high.

Indeed, a better visualization of temperature effects in a reasonable range is obtained from the static conductivity. An illustrative case is that of $U=0.9$ at half-filling, shown in Fig. 11 for various disorder strengths. In the Mott state at low disorder, the conductivity is thermally activated, but this behavior changes to that of a metal as the disorder increases. In this last regime, further growth of disorder intensity causes a reduction of the conductivity and leads to its vanishing in the Anderson-localization regime. Even though it is restricted to a specific interaction value and band filling, Fig. 11 contains the two kinds of behavior observed for the other cases studied.

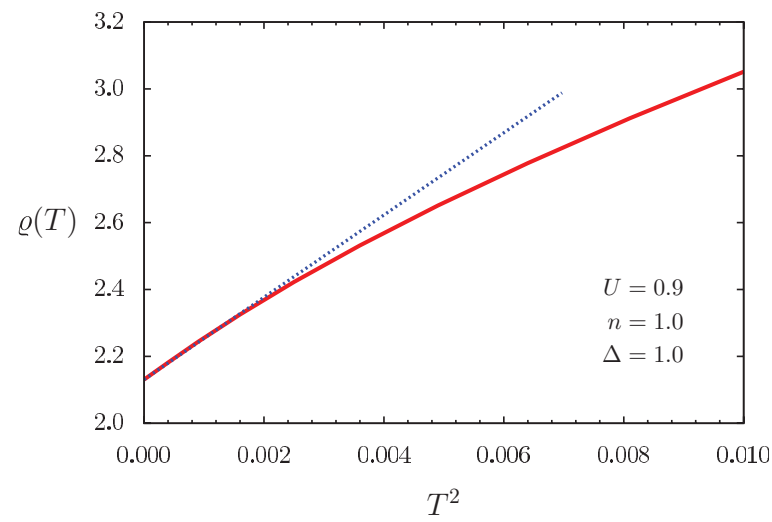

FIG. 12. (Color online) Resistivity in the low-temperature region for a case of a disorder-induced metallic state. Notice that the horizontal axis is the temperature squared. The dotted straight line is just an aid to visualize the initial Fermi-liquid behavior. 
For $U=0.3$, one observes only the metallic behavior, while for $U=1.5$ the behavior is always of the activated type.

It is interesting to point out that plotting the resistivity $\varrho(T)=1 / \sigma(T)$ allows us to see a $T^{2}$ dependence (on top of a finite residual resistivity) at very low temperatures. This is shown in Fig. 12, and it indicates that the metallic regime induced by disorder is of a Fermi-liquid nature. However, this is not the case in the clean limit when a metallic state exists at weak coupling, as we already observed in the optical conductivity for $U=0.3$.

\section{CONCLUSIONS}

We have analyzed the combined effects of Coulomb correlation, local disorder, and band filling using an AndersonFalicov-Kimball model, which means including Coulomb correlations in a simplified version of the Hubbard model without spin-flip, and adding disorder through a uniform distribution of on-site energies. This analysis involved calculation of one-particle Green's functions in the DMFT scheme, with arithmetic and geometric averages over disorder. With this, we obtained the single-particle DOS and built up diagrams showing the spectral distribution of extended and localized states, as well as the presence of correlation gaps for typical interaction or disorder regimes, with special focus on the effects of varying the electron density.

One of our main results is a set of phase diagrams for various band fillings as a function of the controlling parameters of correlation $(U)$ and disorder $(\Delta)$. It shows clearly that a
Mott-insulator state exists only at half-filling. Nevertheless, for densities not far from $n=1$ the presence of a correlation gap, even though it does not contain the Fermi level, is relevant, affecting the nature of the Anderson-localization regime induced at moderate disorder. In this region, the phase diagram shows reentrance of the metallic phase before the final establishment of Anderson localization at strong disorder.

Our study was complemented by calculation of the optical and static conductivities for the various cases, which could be consistently compared with the scenario inferred from the spectral diagrams. Here we obtain that, although the FalicovKimball model with finite Coulomb interaction and no disorder is not a Fermi liquid, the metallic state induced by disorder does show Fermi-liquid behavior in the low-temperature variation of the resistivity.

As a final comment, we would like to stress that, despite the simplification introduced by the Falicov-Kimball model in the description of a true electronic conduction band, the possibility of an exact solution within the DMFT scheme allows for a detailed investigation of the interplay between correlation and disorder. In addition, it is worth mentioning that actual FK systems can be experimentally realized in cold-atom optical lattices, ${ }^{21}$ for which the kind of calculations performed in the present work can be of significant value.

\section{ACKNOWLEDGMENTS}

This work was supported in part by Conselho Nacional de Desenvolvimento Científico e Tecnológico (CNPq), Brazil.
${ }^{1}$ N. Mott and R. Peierls, Proc. R. Soc. London 49, 72 (1937).

${ }^{2}$ J. Hubbard, Proc. R. Soc. London, Ser. A 276, 238 (1963).

${ }^{3}$ P. W. Anderson, Phys. Rev. 124, 41 (1961).

${ }^{4}$ V. Dobrosavljevic, A. A. Pastor, and B. K. Nikolic, Europhys. Lett. 62, 76 (2003).

${ }^{5}$ A. M. C. Souza, D. O. Maionchi, and H. J. Herrmann, Phys. Rev. B 76, 035111 (2007).

${ }^{6}$ W. Metzner and D. Vollhardt, Phys. Rev. Lett. 62, 324 (1989).

${ }^{7}$ A. Georges, W. Kotliar, G. Krauth, and M. Rozemberg, Rev. Mod. Phys. 68, 13 (1996).

${ }^{8}$ D. Vollhardt, Dynamical Mean-Field Theory of Electronic Correlations in Models and Materials, AIP Conference Proceedings No. 1297, edited by A. Avella and F. Mancini (AIP, New York, 2010), p. 339.

${ }^{9}$ R. Bulla, Phys. Rev. Lett. 83, 136 (1999).
${ }^{10}$ P. G. J. van Dongen and D. Vollhardt, Phys. Rev. Lett. 65, 1663 (1990).

${ }^{11}$ L. Falicov and J. Kimball, Phys. Rev. Lett. 22, 997 (1969).

${ }^{12}$ U. Brandt and C. Mielsch, Z. Phys. B 75, 365 (1989).

${ }^{13}$ V. Janis, Z. Phys. B 83, 227 (1991).

${ }^{14}$ J. K. Freericks and V. Zlatić, Rev. Mod. Phys. 75, 1333 (2003).

${ }^{15}$ K. Byczuk, W. Hofstetter, and D. Vollhardt, Phys. Rev. Lett. 94, 056404 (2005).

${ }^{16}$ K. Byczuk, Phys. Rev. B 71, 205105 (2005).

${ }^{17}$ M. A. Gusmão, Phys. Rev. B 77, 245116 (2008).

${ }^{18}$ H. Bethe, Proc. R. Soc. London, Ser. A 150, 552 (1935).

${ }^{19}$ E. MÃ $\frac{1}{4}$ ller-Hartmann, Z. Phys. B 76, 211 (1989).

${ }^{20}$ M. Jarrell, J. K. Freericks, and T. Pruschke, Phys. Rev. B 51, 11704 (1995).

${ }^{21}$ M. M. Maśka, R. Lemański, J. K. Freericks, and C. J. Williams, Phys. Rev. Lett. 101, 060404 (2008). 